\title{
On Congruent Numbers
}

\author{
Alex Nguhi
}

\section{Abstract}

With respect to some classification of Pythagorean triples, if a number $k$ is congruent then it can easily be proven. This expands the quest to resolve the congruent number problem. A proposition is put forward on rational sides forming a congruent number

\section{Introduction}

The congruent number problem is somehow a million-dollar question owing to the fact that Tunnel's Theorem leads to the BirchSwinnerton Conjecture which Clay Mathematics has a cash prize of 1 million dollars in the Millennium Questions [1,2]

An integer number is congruent if it's equal to the area in a rightangled triangle of rational sides,[3].

This paper builds on a previous classification of Pythagorean triples[4]. If we indeed use the Archetypal equations, we can easily deduce faster methods of defining Congruent Numbers obtained by integer sides. 


\section{Statement of Results}

\subsection{Integer Sides}

\subsubsection{Archetype 1 Congruents}

A brief description of Pythagorean triples $a, b, c$ of Archetype 1 is that a :

$a$ is an odd number greater than or equal to 3

$b=\frac{a^{2}-1}{2}$

$c=\frac{a^{2}+1}{2}$

The congruent number can be described as :

$$
\begin{gathered}
k=\frac{1}{2}(a \times b) \\
k=\frac{1}{2}\left(a \times \frac{a^{2}-1}{2}\right) \\
k=\frac{a^{3}-a}{4}
\end{gathered}
$$

Hence $k$ is always a congruent number if $a$ is an odd number. $\mathrm{k}=6,30,85,180, \ldots$

\subsubsection{Archetype $2 \& 3$}

A generalization of Archetype 2 and 3 for sides $a, b, c$ is that :

$c=r^{2}+z$

$b=r^{2}-z$

$a=\sqrt{c^{2}-b^{2}}=\sqrt{4 z r^{2}}=2 r \hat{z}$

here $\hat{z}=\sqrt{z}$ 
Hence to solve for $\mathrm{k}$ :

$$
\begin{gathered}
k=\frac{1}{2}(a \times b \\
k=\frac{1}{2}\left(2 r \hat{z} \times\left(r^{2}-z\right)\right) \\
k=\hat{z}\left(r^{3}-r z\right)=\hat{z}\left(r^{3}-r \widehat{z}^{2}\right) \\
k=r^{3} \hat{z}-r \widehat{z}^{3}
\end{gathered}
$$

As long as $r^{3} \hat{z}>r \widehat{z}^{3}$ we get nice solutions that form Pythagorean triples.

\subsection{Congruent Number from Non-Integer Sides(atleast 1 non-integer side)}

Here the main barrier is that all sides need to be rational. Taking this into account then:

$$
\begin{aligned}
& a=\frac{p_{a}}{q_{a}} \\
& b=\frac{p_{b}}{q_{b}} \\
& c=\frac{p_{c}}{q_{c}}
\end{aligned}
$$

If $k$ is a congruent number then all $p^{\prime}$ s and $q$ 's are integers Using the integers $p$ and $q$ to represent fractions/rational numbers then we can conjecture several important statements from the behaviour.

\section{Hypothesis 1}

if $q_{c}=q_{a} q_{b}$ then $\left(p_{a}, p_{b}, p_{c}\right)$ can be generated from archetypal equations. 


\section{Hypothesis 2}

We can make a generalized version of Hypothesis 1 by letting :

$q_{c}=f\left(q_{a}, q_{b}\right)$, the numerators remain archetypal triples One such function $f$ could an LCM. In such a case Hypothesis 1 holds if either $q_{a}, q_{b}$ is 1 or they are prime to each other (no common factors).

An Analysis of David Golbergs' solutions for rational Pythagorean Triples having the Congruent Property obey Hypothesis 1 [5]

The area that requires rigorous effort is the values of $q_{a} q_{b}$ that completely factorizes a Pythagorean triple in the fashion

$$
\frac{1}{q_{a} q_{b}}(A, B, C)
$$

for $A, B, C$ triple integers corresponding to $\left(p_{a} q_{b}, p_{b} q_{a}, p_{c}\right)$

From this we can dedice that :

i. $C$ is uniquely determined

ii. A,B can never be prime numbers unless in a case where the denominator is 1 .

iii. if $\mathrm{k}$ is a congruent number then :

$$
k=\frac{1}{2 q_{a}^{2} q_{b}^{2}} A B
$$

One major consequence is the following theorem.

Theorem If $(A, B, C)$ is an integer Pythagorean triple with $A$ and $B$ having at least one squared factor for each, then there must be a congruent number $k$ with respect to the square factors.

This Theorem combined with known divisibility methods means we can churn out congruent numbers very fast from non integer sides.

For Archetype 1 as with integers previously, we can right away pick out the congruent number. 
In the regions $9,25,49,81 \ldots$ we can calculate $(\mathrm{A}, \mathrm{B})$ sides $\left(9, \frac{9^{2}-1}{2}\right)=(9,8 \times 5)$

Here $k=5$

$\left(25, \frac{25^{2}-1}{2}\right)=(25,8 \times 39)$

Here $k=39$

$\left(49, \frac{49^{2}-1}{2}\right)=(49,16 \times 75)$

Here $k=150$

A generalization would be that the congruent number depends on the prime factorization of $B$ on the condition none has the integer 1 as a denominator. Otherwise, both A and B determine the congruence.

\section{Archetypes 2 \& 3}

These ones require a combination of sides $A$ and $B$ and also usual square number distribution.

\section{Some unique behavior}

Consider the congruent number formed by the triple $(9,40,41)$ ie 180

After complete factorization $4 \times 5 \times 9$. From the rationals sides with at least 1 non-integer side we have 5,45 , as congruent numbers.

This behavior can also be extended to other triples.

\section{Conclusion}

Congruent numbers can described using archetypal equations 


\section{References}

[1]. Koblitz N. Introduction to Elliptic Curves and Modular Forms- Graduate Texts in Mathematics. 97 (2nd ed.). SpringerVerlag. - 320 p.

[2]. Carlson, J. A., Jaffe, A., \& Wiles, A. (2006). The millennium prize problems. Cambridge, MA, American Mathematical Society, Providence, RI: Clay Mathematics Institute.

[3]. Top, J., \& Yui, N. (2008). Congruent number problems and their variants. Algorithmic Number Theory, 44, 613-639.

[4]. Nguhi, A., \& Kweyu, C. (2021). On the Pythagorean Triples' Equations and the Perfect Cuboid Problem. OSF Preprints. April, 4.

[5]. Goldberg, D. (2021). Triangle Sides for Congruent Numbers less than 10,000. arXiv preprint arXiv:2106.07373. 